\title{
SHINING 3D EINSCAN-PRO, APPLICATION AND VALIDATION IN THE FIELD OF CULTURAL HERITAGE, FROM THE CHILLIDA-LEKU MUSEUM TO THE ARCHAEOLOGICAL MUSEUM OF SARNO
}

\author{
S. Morena ${ }^{1, *}, \mathrm{~S}$. Barba $^{1}$, A. Álvaro-Tordesillas ${ }^{2}$ \\ ${ }^{1}$ Dept. of Civil Engineering, University of Salerno, Italy - (smorena, sbarba)@unisa.it \\ ${ }^{2}$ Dept. of Urban Planning and Architecture Representation, University of Valladolid, Spain - tordesillas@arq.uva.es
}

Technical Commission II

KEY WORDS: Low cost, Hand-held, Structured light, Comparison, Heritage, Sculpture, Museum

\begin{abstract}
:
The following contribution focuses on the low-cost Shining 3D EinScan-Pro scanner, above all the analysis of its precision and accuracy. The need to prove the functioning of this instrumentation in practical cases (the sculptures by Eduardo Chillida preserved in the Chillida-Leku Museum and along with some artefacts collected in the Archaeological Museum of Sarno), has led to the comparison and validation of the instrument through a methodology necessarily diversified from the guideline VDI/VDE 2634, part 2 and part 3, characteristics to the test the optical 3D measuring systems with planar measurement, which works according to the triangulation principle. In particular, two types of comparisons were made: geometric-formal and metric-dimensional. The first type of analysis was carried out analysing the geometric parameters of the models, suitable for validating the information: dimensional (difference between some main measurements); superficial (total mesh extension) and of the form (that is, the discrepancies returned through a DEM analysis). The second type of analysis, instead, of the metric type, was carried out. The complete results of the various analyses will be presented and critically discussed within this contribution in order to prove the stability and the metric quality of this hand-held EinScan-Pro, following the comparison with medium-high end systems now well established in the field of cultural heritage survey.
\end{abstract}

\section{INTRODUCTION}

Reverse Modelling in the field of cultural heritage is nowadays increasingly implemented: conservation and valorisation, in fact, require both digital and physical documentation, to investigate, the 'historical' evolution of forms and colour. The generation of the three-dimensional models, ensures the storage of data in virtual databases, and makes the findings available for consultation even remotely, at the same time, it is an element of protection for evidence to be safeguarded and preserved. Another interesting aspect is the possibility to conduct non-destructive tests and simulations on the models; that is a practice not only widespread in the field of cultural heritage (Farina et al, 2019)

Currently, a significant contribution in the generation of increasingly metrically correct models has come from the great development of hand-held scanners, now more than ever present on the market, each with a different level of precision and accuracy. The similar progress of the software component has made the pursuit of increasingly ambitious objectives possible, aiming to achieve stability and metric quality also through economical instruments. The application of structured light technologies is widely used in the cultural heritage survey, in particular for the $3 \mathrm{D}$ documentation of medium and small objects in which the absence of contact is a fundamental requirement (Akca et al., 2006). Also on the basis of past studies conduct on the survey of sculptures with non-uniform textures, non-reflective colours and with poorly elaborated shapes with the implementation of low-cost technologies (Morena et al., 2014), further test has been carried out on what the expectations in the use of such and new instruments applied to the survey of the sculptural heritage could be to date. In this case, the EinScan-Pro scanner of the Shining 3D company - with an industrial version (tripod and turntable) - was the instrument object of experiment. The case studies on which this hand-held technology was tested are two sculptures by Eduardo Chillida preserved in the Chillida-Leku Museum of Hernani: "Lurrak" $(28 \times 28 \times 23 \mathrm{~cm})$ in terracotta and "Ilarik II" $(141 \times 21.4 \times 19.2$ $\mathrm{cm}$ ) in wood (Garcìa Marcos, 2005); in addition two other preserved finds in the Archaeological Museum of Sarno: an "Olla" of the tomb 336 (VI century BC) and a "Statuette" of the female tomb 769 (IV century BC), both in terracotta (Figure 1). To support the verification of these tools implemented the scanners Artec Eva and Spider, of the Artec3D Company were used, as well as, the implementation of the photogrammetric paradigm with Nikon D3200 SRL camera. For empirical verification, it was necessary to identify two types of comparisons; one type was geometricformal and the other metric-dimensional. These are different to the guideline VDI/VDE 2634, part 2 and part 3, characteristic for the testing of optical 3D measuring systems with planar measurement (kersten, 2016; Boehm, 2016). This paper, therefore, will present an analysis of the results for comparing different instruments in order to prove the validity of the acquisition with the EinScan-Pro instrument, for a first assessment of reliability and quality in the case of acquisitions of small and medium-sized objects in museums.

* Corresponding author: smorena@unisa.it 


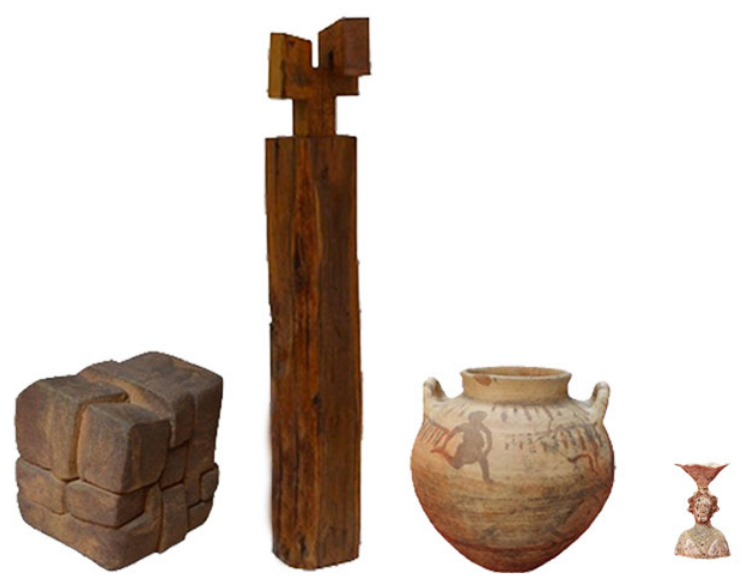

Figure 1. Sculpture and evidences surveyed at the Chillida-Leku Museum (left) and Archaeological Museum of Sarno (right)

\section{MAIN CHARACTERISTICS OF THE INSTRUMENTS USED}

The data acquired, therefore, using different instruments (Figure 2) and post-processed with the software described below; (all the information on the showed characteristics are based on what is contained in the relevant manuals).

\subsection{EinScan-Pro}

User-friendly 3D Shining scanner for surveying medium-sized objects; comes in a modular design, which supports up to four scanning modes: handheld rapid, HD, fixed or with turntable. Based on the projection of white LEDs, it is equipped with a central projector and 2 side monochrome cameras (located at the extremity of the instrument) as well as an additional 1.3 MP external camera (in the colour pack version) for the acquisition of colorimetric information. It allows resolutions up to 0.16 $\mathrm{mm}$, accuracy of $0.05 \mathrm{~mm}$ and acquisitions with a maximum speed of 15 fps (Jain and Zhang; 2019).

The scanner's proprietary software is the EinScan 2.0 with a simple and intuitive interface. The alignment algorithm, however, is slightly limiting as it can detect manually a maximum number of three homologous points between the various scans. In addition, the restricted options for managing the model (clean, fusion, close holes, measurements, etc.) necessarily involves the implementation of third-party software (which in itself does not represent a real limit, in the case of applications for research purposes). A limit, instead, is the possibility to implement the software, for the data processing phase, only in the presence of scanners and optional gadgets connected to the computer, which makes the procedure slightly more cumbersome and complex.

\subsection{Artec Eva and Spider}

Produced by the Artec 3D, the Artec Eva is a triangulation scanner with structured light (flash bulb) ideal for prompt acquisition of objects also with medium size. The maximum resolution achievable is $0.5 \mathrm{~mm}$, with an accuracy of $0.1 \mathrm{~mm}$ acquiring up to $16 \mathrm{fps}$. The range of the linear field of view ranges from a minimum of $214 \times 148 \mathrm{~mm}$ to a maximum of $536 \times 371 \mathrm{~mm}$ and the camera available for the acquisition of colorimetric information is $1.3 \mathrm{MP}$.
To same manufacturer, the Artec Spider is also a structured light scanner (speckle pattern) based on blue LED technology more suitable, however, for the detection of objects of small size and/or complex surface. It allows a maximum resolution of $0.1 \mathrm{~mm}$ and an accuracy of $0.05 \mathrm{~mm}$, to acquire at a maximum speed of $7.5 \mathrm{fps}$. The scanner works in a linear field of view between $90 \times 70 \mathrm{~mm}$ and $180 \times 140 \mathrm{~mm}$ with the colorimetric information is obtained, once again, by means of a 1.3 MP camera. Artec Studio Professional - implemented in the release 11 - is the scanner's proprietary software, with an intuitive and very practical interface that allows good management of the settings by the operator in order to conduct all the different phases of the post-process. Compared to the previous software, it also allows a good interaction for the correction and decimation of the mesh, as well as, the ability to coordinate the phase of texturization, to choose between the optional triangle map or texture atlas, and possibly to make analysis and measurements on the models (applications of these instruments have been published by Wang et al., 2018; Inzerillo et al., 2015 and Adams et al., 2015).

\subsection{Nikon D3200}

This entry-level digital camera and single-lens reflex (dSLR) is equipped with a CMOS sensor $(23.2 \times 15.4 \mathrm{~mm})$ of $24.2 \mathrm{MP}$ for image of $6016 \times 4000$ pixels. In production from 2012 to 2014, it was characterized by the new EXPEED3 image-processing engine adept to generate photos with high colour quality and low noise at high resolutions (Hess, 2012).

The choice of the photogrammetric software implemented fell on the SfM (Structure for Motion) algorithm of the Agisoft, which, as is well known, can perform photogrammetric elaborations in a pseudo-automatic way for the generation of photo-realistic three-dimensional models. It is common to observe that, different from the previous applications, the photogrammetric paradigm does not directly generate a model in real shape and size, which is why it was necessary to scale based on previously acquired three-dimensional metric information.

\section{DATA ACQUISITION}

The acquisitions of the sculptures made in the above-mentioned museums, that is, in contexts completely different from ad hoc laboratories and, therefore, subject to certain limitations for the control of space and light: sculptures close walls or high exposures and non-uniform lighting.

In the case of hand-held scanners, the instrumentation is rotated around the entire object with slow and uniform movements. The data collected can be monitored in real time on the notebook and displayed by the proprietary software. Sudden movements could cause a signal loss; a (frequent) problem that is most often could be handled during post-processing phase.

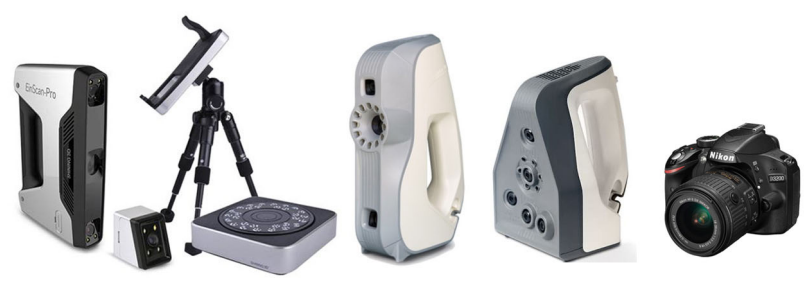

Figure 2. From left to right: Einscan-Pro, Artec Eva, Artec Spider, Nikon D3200 
These techniques make rapid acquisitions possible allowing you to detect, in a day a large amount of sculptures and data, generally, it was usually a few minutes per scan; it depends on the size of the object and its geometric and colorimetric properties. As is well known, in fact, structured light scanners are quite influenced by the surface optical characteristics of objects, with some limitations in the acquisition of shiny, transparent, reflective or dark coloured materials (Georgopoulos, Ioannidis, 2010).

In the case of the image-base technique, once again, the acquisitions have been obtained, by rotating around the entire object, operating with a constant distance and a fixed focal length, as well as, trivially, guaranteeing a sufficient overlap between one shot to the next. The various case studies surveyed are listed below.

"Lurrak": given the medium size of the sculpture $(28 \times 28 \times 23$ $\mathrm{cm}$ ), its opaque texture, as well as, its geometric characteristics, the acquisitions with the Artec Eva scanner were resolved quickly enough. The scans acquired in geometry and texture mode were processed with Artec Studio's proprietary software, to ensure the creation of a mesh model characterized by few holes and valuable colorimetric information.

The sculpture was also detected with the EinScan-Pro scanner, however, it obtaining significant gaps on the model. The six scans acquired on-site (geometry and texture) were progressively and automatically aligned by the EinScan 2.0 software, while the next phase of mesh management was conducted in Artec Studio. A final survey of Chillida's work was conducted with the Nikon D3200 to operate with an ISO 200, $32 \mathrm{~mm}$ focal length and f/14 aperture (Laurențiu-Marian, 2019). In this case, the data was processed in Agisoft.

"Ilarik II": wooden structure $(141 \times 21.4 \times 19.2 \mathrm{~cm})$ measured, once again, with Artec Eva and EinScan-Pro, but operating, in the latter case, with an algorithm of only geometry. During the acquisition phase, due to homogeneous texture and poorly articulated geometry, the instrumentation lost the signal several times, which is why it was decided to operate the scan by acquiring 11 separate scans (covering the entire sculpture), aligning them, only in the second phase, with the application Artec Studio.

"Olla" belonging to the tomb 336: made of terracotta, the archaeological find was acquired with both Artec Eva and EinScan-Pro; as in the previous case, the three scans (acquired in geometry only mode) were then aligned and textured in Artec Studio.

"Statuetta" belonging to the female tomb 769: also in terracotta, but given its small size, it was surveyed with both the Artec Spider and the EinScan-Pro scanner, working in both cases with the use of a turntable. To proceed in automatic mode, the EinScan-Pro was placed on a tripod at about $400 \mathrm{~mm}$ from the turntable, with an angle of inclination such as to cover the entire object. Thanks to the simple geometry, the light colour and the porous material of the object, the acquisition phase was not difficult, 5 scans were returned to be automatically aligned by the proprietary software.

\section{COMPARISON}

The geometric-formal and metric-dimensional evaluation data was carried out with the aid of Geomagic Studio software - in the release 2013 -, in which case the different lengths were measured and recorded, and used Artec Studio for the realization of DEM analysis.

\subsection{Geometric-formal analysis}

The purpose of this analysis is to highlight any differences in measurements (DS), surfaces (DSh) and form (DF) between the models acquired with the various technologies.

In particular, in the case of DS, seven characteristic measurements were recorded for each sculpture/archaeological evidence, with DSh, on the other hand, the difference of polygons that compose the model mesh was evaluated and, lastly, with DF, a DEM analysis to evaluate the variances and deviations of the three-dimensional models.

The data shown in the tables and illustrated in the graphs below correspond to the difference that exist between the measurements of the EinScan-Pro models compared to the Artec scanners (Eva or Spider in relation to the size of the object). We proceed below to analyse individually the punctual data obtained from the comparison

4.1.1 "Lurrak": the two models compared show, basically, differences previously highlighted. The main problems found are related to the geometry with the presence of marked coves that generate shadow beyond the dark and homogeneous colour. The presence of gaps on the model of EinScan-Pro and the difficulty in identifying natural targets for the particular homogeneous texture of the object have inevitably led to a high number of differences close to $1 \mathrm{~mm}$ (Table 1 and Figure 3) compared to the model Artec Eva (reference), as well as, an incomplete 3D model also found in the limited number of polygons that compose the mesh (Table 2).

The DEM analysis carried out with the DF analysis showed what has already been reported by DS, deviations of the surface in absolute value less than $1 \mathrm{~mm}$ (blue and yellow areas) and high information gaps (in grey) mainly in the inlets and in the upper and lower part of the model (Figure 4).

\begin{tabular}{|c|c|c|c|}
\hline \multicolumn{4}{|c|}{ Lurrak (DS) } \\
\hline & $\begin{array}{c}\text { Artec Eva } \\
{[\mathbf{m m}]}\end{array}$ & $\begin{array}{c}\text { EinScan-Pro } \\
{[\mathbf{m m}]}\end{array}$ & $\begin{array}{c}\text { Deviation } \\
{[\mathbf{m m}]}\end{array}$ \\
\hline Linear1 & 52.06 & 51.21 & 0.85 \\
\hline Linear2 & 57.82 & 57.96 & -0.14 \\
\hline Linear3 & 80.85 & 80.96 & -0.11 \\
\hline Linear4 & 86.50 & 86.32 & 0.18 \\
\hline Linear5 & 87.31 & 88.30 & -0.99 \\
\hline Linear6 & 97.40 & 97.58 & -0.18 \\
\hline Linear7 & 99.61 & 98.72 & 0.89 \\
\hline
\end{tabular}

Table 1. DS analysis of the "Lurrak" model

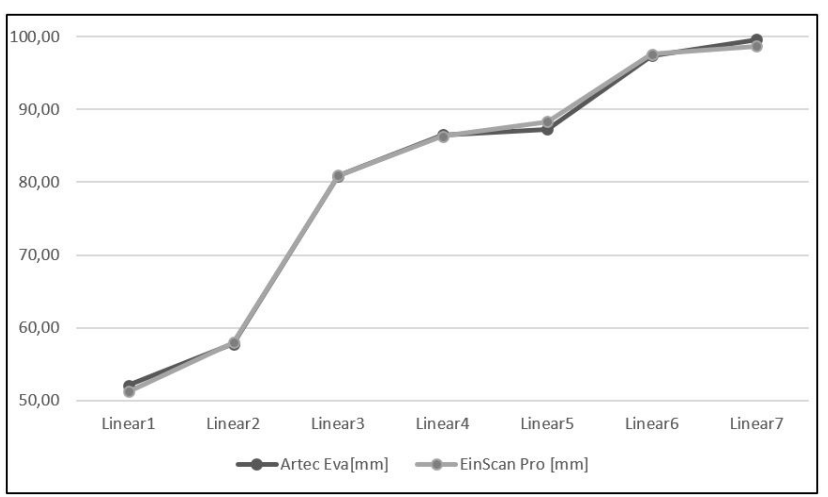

Figure 3. Graph of the DS analysis of the "Lurrak" model 


\begin{tabular}{|c|c|c|c|}
\hline \multicolumn{4}{|c|}{ Lurrak (DSh) } \\
\hline & Artec Eva & EinScan-Pro & Deviation \\
\hline Mesh & 1126448 & 1077333 & 49115 \\
\hline
\end{tabular}

Table 2. DSh analysis of the "Lurrak" model
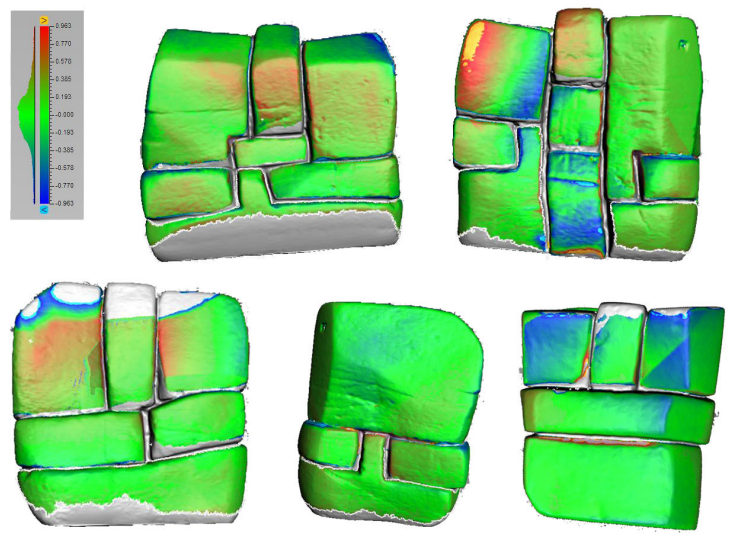

Figure 4. DF analysis of the "Lurrak" model

4.1.2 "Ilarik II": the two models have significant divergences reaching differences of more than $1 \mathrm{~mm}$ (Table 3 and Figure 5). They are mainly found at the top of the model, where the geometry of the sculpture is more complex. During the acquisition phase with EinScan-Pro, in fact, the scanner has found a series of problems with consequent loss of signal. The need to integrate more scans, moreover, has involved the generation of a model with a greater number of polygons (Table 4), as well as, a post-processing phase in a third-party software that has involved a greater alteration of the final model. From the DEM analysis (Figure 6) it emerges that the surfaces of the two models are distant, in many points, with greater deviations in the upper part (blue-yellow zone) with values greater than 1 $\mathrm{mm}$, confirming what was previously exposed.

\begin{tabular}{|c|c|c|c|}
\hline \multicolumn{5}{|c|}{ Ilarik II (DS) } \\
\hline & $\begin{array}{c}\text { Artec Eva } \\
{[\mathbf{m m}]}\end{array}$ & $\begin{array}{c}\text { EinScan-Pro } \\
{[\mathbf{m m}]}\end{array}$ & $\begin{array}{c}\text { Deviation } \\
{[\mathbf{m m}]}\end{array}$ \\
\hline Linear1 & 59.87 & 58.65 & 1.22 \\
\hline Linear2 & 74.89 & 73.41 & 1.48 \\
\hline Linear3 & 126.71 & 128.08 & $-1,32$ \\
\hline Linear4 & 136.46 & 136.11 & 0.35 \\
\hline Linear5 & 149.77 & 149.95 & -0.18 \\
\hline Linear6 & 141.05 & 140.96 & 0.09 \\
\hline Linear7 & 212.62 & 212.93 & -0.31 \\
\hline
\end{tabular}

Table 3. DS analysis of the "Ilarik II" model

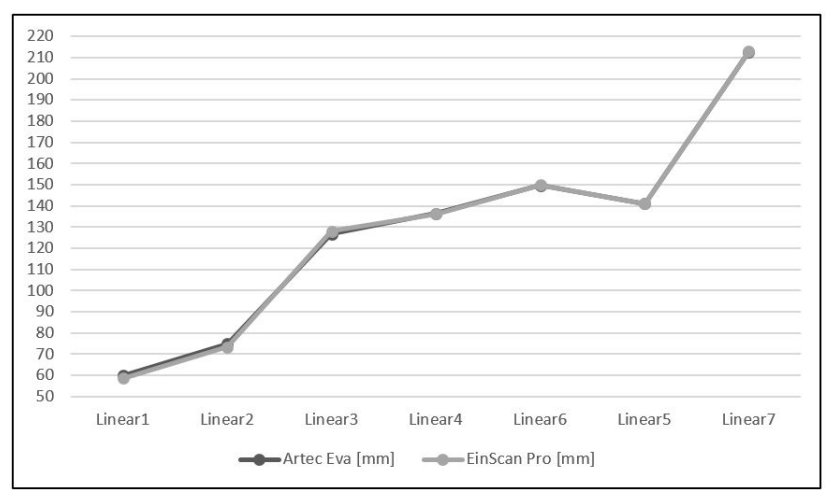

Figure 5. Graph of DS analysis of "Ilarik II" model

\begin{tabular}{|c|c|c|c|}
\hline \multicolumn{4}{|c|}{ Ilarik II (DSh) } \\
\hline & Artec Eva & EinScan-Pro & Deviation \\
\hline Mesh & 2393180 & 2393278 & -98 \\
\hline
\end{tabular}

Table 4. DSh analysis of the "Ilarik II" model
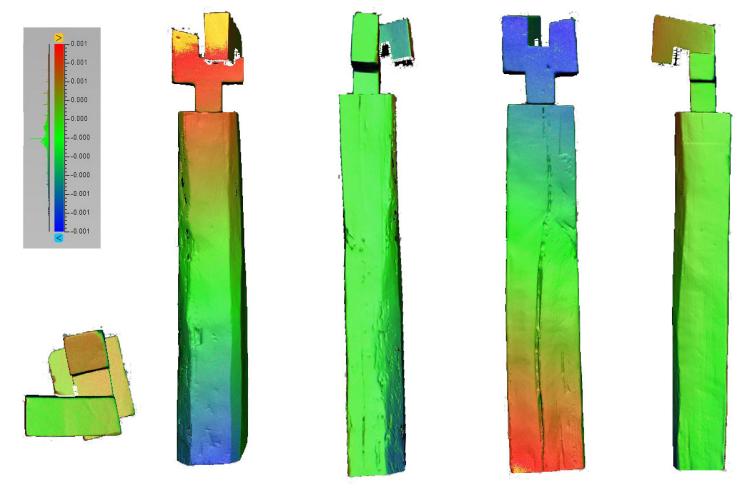

Figure 6. DF analysis of the "Ilarik II" model

"Olla" belonging to the tomb 336: despite a series of difficulties in the acquisition phase, the result is presented with a well textured and modelled. As deduced from the DS analysis (Table 5) all the measurements have values lower than $1 \mathrm{~mm}$ despite the difficulty of survey of the measurements based mainly on natural targets found on the texture rather than on the geometry of the object. The DSh analysis has shown, once again, a greater number of polygons than that of Artec, as in the previous case, in fact, it was necessary to divide the survey into several parts to proceed to assemble them later with some redundancy of information (Table 6). The DF analysis, as shown in the figure 8 , has revealed, once again, that a significant number of points on the surface have deviations of less than $1 \mathrm{~mm}$, with greater deviation near the upper part where the geometry is slightly more complex.

\begin{tabular}{|c|c|c|c|}
\hline \multicolumn{4}{|c|}{ "Olla" (DS) } \\
\hline & $\begin{array}{c}\text { Artec Eva } \\
{[\mathbf{m m}]}\end{array}$ & $\begin{array}{c}\text { EinScan-Pro } \\
{[\mathbf{m m}]}\end{array}$ & $\begin{array}{c}\text { Deviation } \\
{[\mathbf{m m}]}\end{array}$ \\
\hline Linear1 & 68.57 & 68.60 & -0.03 \\
\hline Linear2 & 97.80 & 97.78 & -0.02 \\
\hline Linear3 & 98.84 & 98.80 & 0.04 \\
\hline Linear4 & 108.82 & 108.93 & -0.11 \\
\hline Linear5 & 114.87 & 114.46 & -0.41 \\
\hline Linear6 & 181.63 & 175.27 & 0.03 \\
\hline Linear7 & 199.72 & 199.34 & 0.38 \\
\hline
\end{tabular}

Table 5. DS analysis of the "Olla" model

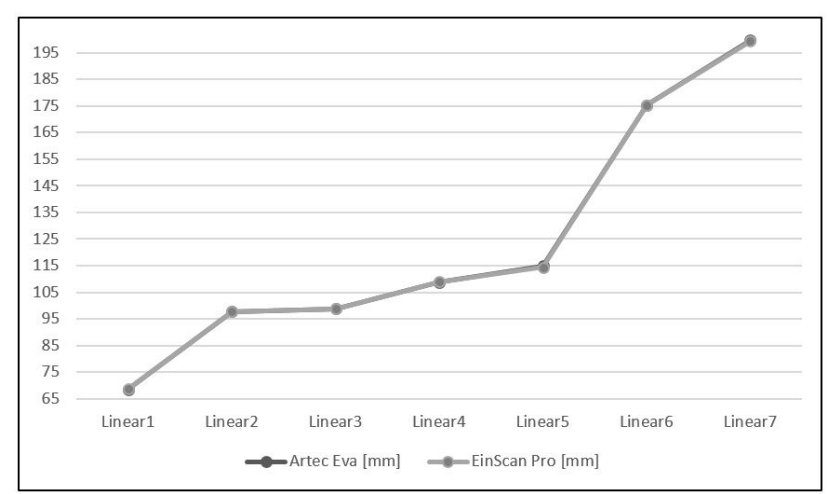

Figure 7. Graph of the DS analysis of the "Olla" model 


\begin{tabular}{|c|c|c|c|}
\hline \multicolumn{4}{|c|}{ "Olla" (DSh) } \\
\hline & Artec Eva & EinScan-Pro & Deviation \\
\hline Mesh & 56426 & 894753 & -838327 \\
\hline
\end{tabular}

Table 6. DSh analysis of the "Olla" model
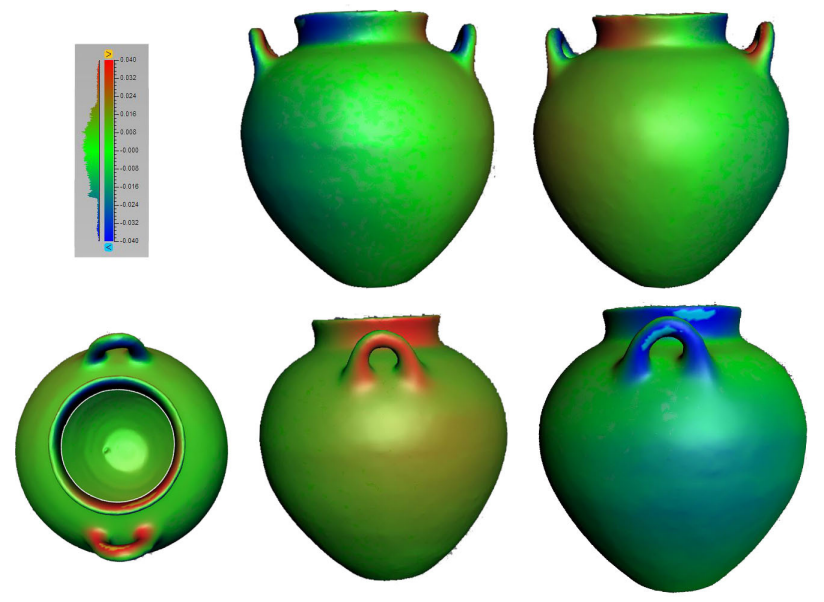

Figure 8. DF analysis of "Olla" model

"Statuette" belonging to the female tomb 769: having worked with a turntable, the accuracy obtained is greater than the other case studies. EinScan-Pro, in fact, returns a complete model with values in the maximum DS analysis of $0.46 \mathrm{~mm}$ (Table 7 and Figure 9), also confirmed by the DF analysis, (Figure 10) in which all the points maintain a distance from the reference surface less than $1 \mathrm{~mm}$ (red and blue) and with many points close to 0 (green). However, from the DSh comparison it can be noticed how the number of polygons is too high compared to the Artec Spider model, this happens because it was necessary to assemble more scans between them, to result in a model heavier than the Artec, and, maybe, less manageable (Table8).

\begin{tabular}{|c|c|c|c|}
\hline \multicolumn{5}{|c|}{ "Statuetta" (DS) } \\
\hline & $\begin{array}{c}\text { Artec Spider } \\
{[\mathbf{m m}]}\end{array}$ & $\begin{array}{c}\text { EinScan-Pro } \\
{[\mathbf{m m}]}\end{array}$ & $\begin{array}{c}\text { Deviation } \\
{[\mathbf{m m}]}\end{array}$ \\
\hline Linear1 & 28.97 & 28.77 & 0.20 \\
\hline Linear2 & 43.79 & 44.08 & -0.29 \\
\hline Linear3 & 51.51 & 51.55 & -0.04 \\
\hline Linear4 & 58.65 & 58.22 & 0.43 \\
\hline Linear5 & 58.72 & 58.98 & -0.26 \\
\hline Linear6 & 64.09 & 63.95 & 0.14 \\
\hline Linear7 & 151.43 & 150.97 & 0.46 \\
\hline
\end{tabular}

Table 7. DS analysis of the "Statuetta" model

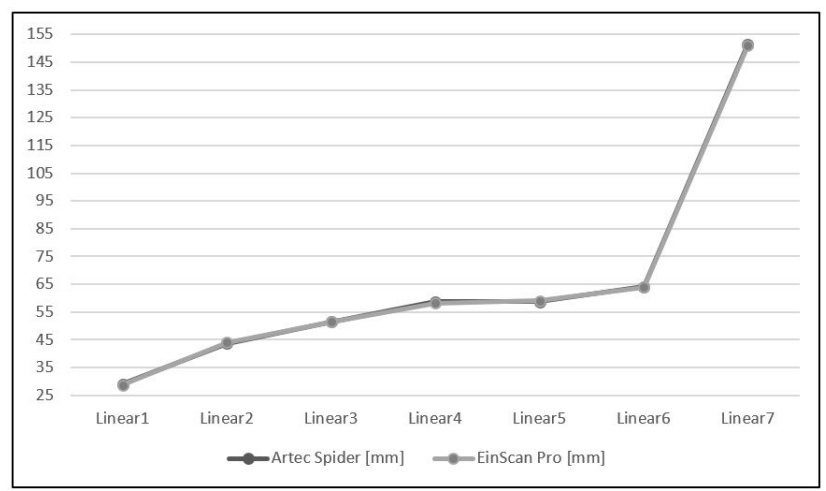

Figure 9. Graph of the analysis DS of the "Statuette" model

\begin{tabular}{|c|c|c|c|}
\hline \multicolumn{5}{|c|}{ "Statuetta" (DSh) } \\
\hline & Artec Eva & EinScan-Pro & Deviation \\
\hline Mesh & 885361 & 3344339 & -2458978 \\
\hline
\end{tabular}

Table 8. DSh analysis of the "Statuetta" model
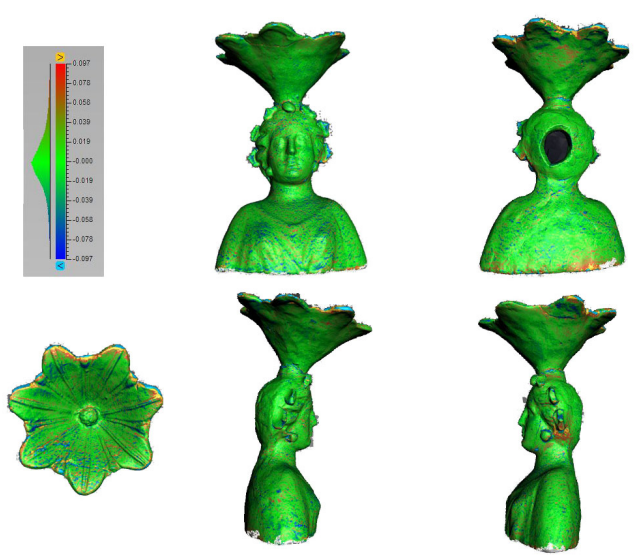

Figure 10. DF analysis of "Statuetta" model

\subsection{Metric-dimensional analysis}

This second type of analysis and comparison between models of the same sculpture detected with multiple tools, finds its basis in the errors theory. The comparison has been realized, for the sculpture "Lurrak" of the Chillida-Leku Museum, to be for its complex form and homogeneous texturization of particular interest. The experiment involved the estimation of the threedimensional distribution of errors, to prove an absolute analysis of the depth of the meshes obtained by the scanner and photogrammetric survey compared to the model of the Artec Eva. Specifically, the three models were imported into Geomagic Studio 2013, aligned respect to the same reference system and, so, the spatial coordinate deviations of 20 points were identified on the evaluated models. The application of the error theory, therefore, in our case consist in a punctual analysis; the comparisons were made at first between the models EinScan-Pro and Arte EVA and, successively, between the scanner models and the photogrammetric one.

We proceeded to calculate some statistical data necessary for comparison, first of all the arithmetic mean necessary to evaluate the difference from the average of the visible coordinates in Table 9, 10 and 11 and, subsequently, the standard deviation $(\sigma)$ necessary to express the precision of the individual measurements. The value $\sigma$, in fact, allows to determine the maximum limit beyond which in practice the errors of a set of measures can no longer be accepted as accidental. Experience shows that $70 \%$ of errors do not exceed the value $\sigma, 95 \%$ do not exceed twice the value $\sigma$ and about $100 \%$, about the totally errors, do not exceed three times the value $\sigma$ defined, therefore, as the maximum tolerable error or tolerance (Barba, Mage, 2014; Hayes, 1998).

In the first comparison, the resulting difference between the coordinates of the 3 points used to reference the models never exceeds the value of $1.06 \mathrm{~mm}$ (Table 12), thus it has been taken as a limit error $\left(\sigma_{\mathrm{EA}}\right)$ for the subsequent analysis of absolute errors. Then, the standard deviation of the remaining points were determined where it is possible to observe, that only $95 \%$ of the values are characterized by tolerable deviations and in particular $81.7 \%$ of the deviations is lower than $\sigma_{\mathrm{EA}}, 13.3 \%$ is between $\sigma_{\mathrm{EA}}$ and $3 \sigma_{\mathrm{EA}}$ (in yellow), while $5 \%$ exceeds the tolerance (in red). 


\begin{tabular}{|c|c|c|c|}
\hline \multicolumn{5}{|c|}{ Coordinates Artec Eva [mm] } \\
\hline Points & $\mathrm{X}, \mathrm{A}$ & $\mathrm{y}, \mathrm{A}$ & $\mathrm{Z}, \mathrm{A}$ \\
\hline $\mathbf{1}$ & 239.92 & 193.16 & 28.03 \\
\hline $\mathbf{2}$ & 178.54 & 184.3 & -24.41 \\
\hline $\mathbf{3}$ & 232.34 & 135.36 & 77.55 \\
\hline $\mathbf{4}$ & 125.32 & 114.95 & -119.22 \\
\hline $\mathbf{5}$ & 92.86 & 70.25 & -141.65 \\
\hline $\mathbf{6}$ & 124.75 & 9.78 & -84.34 \\
\hline $\mathbf{7}$ & 240.86 & 10.78 & 49.82 \\
\hline $\mathbf{8}$ & 119.06 & 211.67 & -33.64 \\
\hline $\mathbf{9}$ & 114.69 & 234.71 & 112.8 \\
\hline $\mathbf{1 0}$ & 30.27 & 120.06 & -123.9 \\
\hline $\mathbf{1 1}$ & -13.85 & 22.01 & -90.1 \\
\hline $\mathbf{1 2}$ & -21.7 & 111.78 & -84.3 \\
\hline $\mathbf{1 3}$ & -84.75 & 146.9 & -12.44 \\
\hline $\mathbf{1 4}$ & -95.7 & -2.84 & 18.36 \\
\hline $\mathbf{1 5}$ & -63.22 & 164.58 & 42.18 \\
\hline $\mathbf{1 6}$ & -17.63 & 22.88 & 134.05 \\
\hline $\mathbf{1 7}$ & 0.84 & 117.06 & 101.86 \\
\hline $\mathbf{1 8}$ & 75.46 & 173.12 & 157.54 \\
\hline $\mathbf{1 9}$ & 83.17 & 66.59 & 198.19 \\
\hline $\mathbf{2 0}$ & 159.5 & -5.25 & 163.89 \\
\hline
\end{tabular}

Table 9. Coordinates of the 20 points of Artec Eva model

\begin{tabular}{|c|c|c|c|}
\hline \multicolumn{4}{|c|}{ Coordinates EinScan-Pro [mm] } \\
\hline Points & $\mathrm{X}, \mathrm{E}$ & $\mathrm{Y}, \mathrm{E}$ & $\mathrm{Z}, \mathrm{E}$ \\
\hline $\mathbf{1}$ & 241.42 & 194.09 & 27.18 \\
\hline $\mathbf{2}$ & 180.02 & 185.22 & -25.13 \\
\hline $\mathbf{3}$ & 232.23 & 135.34 & 77.48 \\
\hline $\mathbf{4}$ & 125.45 & 114.96 & -119.38 \\
\hline $\mathbf{5}$ & 93.67 & 70.59 & -142.56 \\
\hline $\mathbf{6}$ & 126.02 & 10.33 & -85.78 \\
\hline $\mathbf{7}$ & 241.88 & 10.82 & 49.28 \\
\hline $\mathbf{8}$ & 119.88 & 212.89 & -35.35 \\
\hline $\mathbf{9}$ & 115.6 & 236.91 & 110.84 \\
\hline $\mathbf{1 0}$ & 29.71 & 119.99 & -124.78 \\
\hline $\mathbf{1 1}$ & -14 & 21.97 & -90.25 \\
\hline $\mathbf{1 2}$ & -22.35 & 111.71 & -85.08 \\
\hline $\mathbf{1 3}$ & -85.74 & 146.79 & -13.73 \\
\hline $\mathbf{1 4}$ & -95.67 & -2.86 & 18.46 \\
\hline $\mathbf{1 5}$ & -63.48 & 164.58 & 42.39 \\
\hline $\mathbf{1 6}$ & -17.09 & 22.6 & 133.63 \\
\hline $\mathbf{1 7}$ & 0.62 & 117.13 & 102.03 \\
\hline $\mathbf{1 8}$ & 75.03 & 173.21 & 157.96 \\
\hline $\mathbf{1 9}$ & 83.35 & 66.66 & 198.37 \\
\hline $\mathbf{2 0}$ & 159.62 & -5.37 & 164.06 \\
\hline
\end{tabular}

Table 11. Coordinates of the 20 points model EinScan-Pro

\begin{tabular}{|c|c|c|c|}
\hline \multicolumn{4}{|c|}{ Coordinates Nikon D3200 [mm] } \\
\hline Points & $\mathrm{X}, \mathrm{N}$ & $\mathrm{y}, \mathrm{N}$ & $\mathrm{Z}, \mathrm{N}$ \\
\hline $\mathbf{1}$ & 237.47 & 192.2 & 28.39 \\
\hline $\mathbf{2}$ & 175.85 & 183.19 & -23.81 \\
\hline $\mathbf{3}$ & 230.42 & 134.39 & 77.64 \\
\hline $\mathbf{4}$ & 124.22 & 114.95 & -117.96 \\
\hline $\mathbf{5}$ & 93.71 & 70.6 & -142.59 \\
\hline $\mathbf{6}$ & 126.82 & 10.47 & -86.58 \\
\hline $\mathbf{7}$ & 241.38 & 10.88 & 49.49 \\
\hline $\mathbf{8}$ & 111.15 & 212.35 & -25.23 \\
\hline $\mathbf{9}$ & 115.33 & 230.06 & 110.96 \\
\hline $\mathbf{1 0}$ & 30.61 & 120.09 & -123.6 \\
\hline $\mathbf{1 1}$ & -13.22 & 21.99 & -89.53 \\
\hline $\mathbf{1 2}$ & -21.65 & 111.84 & -84.26 \\
\hline $\mathbf{1 3}$ & -83.96 & 146.37 & -12.83 \\
\hline $\mathbf{1 4}$ & -94.46 & -3.24 & 19.24 \\
\hline $\mathbf{1 5}$ & -62.63 & 164.03 & 41.72 \\
\hline $\mathbf{1 6}$ & -13.76 & 20 & 129.52 \\
\hline $\mathbf{1 7}$ & 1.8 & 116.3 & 100.64 \\
\hline $\mathbf{1 8}$ & 75.55 & 172.86 & 157.44 \\
\hline $\mathbf{1 9}$ & 86.05 & 64.65 & 195.13 \\
\hline $\mathbf{2 0}$ & 159.31 & -5.62 & 162.46 \\
\hline
\end{tabular}

Table 10. Coordinates of the 20 points of Nikon D3200 model

\begin{tabular}{|c|c|c|c|}
\hline \multicolumn{5}{|c|}{ EinScan-Pro - Artec $[\mathbf{m m}]$} \\
\hline Points & $\sigma_{\mathrm{x}, \mathrm{EA}}$ & $\sigma_{\mathrm{y}, \mathrm{EA}}$ & $\sigma_{\mathrm{z}, \mathrm{EA}}$ \\
\hline $\mathbf{1}$ & 1.06 & 0.66 & 0.60 \\
\hline $\mathbf{2}$ & 1.05 & 0.65 & 0.51 \\
\hline $\mathbf{3}$ & 0.08 & 0.01 & 0.05 \\
\hline $\mathbf{4}$ & 0.09 & 0.01 & 0.11 \\
\hline $\mathbf{5}$ & 0.57 & 0.24 & 0.64 \\
\hline $\mathbf{6}$ & 0.90 & 0.39 & 1.02 \\
\hline $\mathbf{7}$ & 0.72 & 0.03 & 0.38 \\
\hline $\mathbf{8}$ & 0.58 & 0.86 & 1.21 \\
\hline $\mathbf{9}$ & 0.64 & 1.56 & 1.39 \\
\hline $\mathbf{1 0}$ & 0.40 & 0.05 & 0.62 \\
\hline $\mathbf{1 1}$ & 0.11 & 0.03 & 0.11 \\
\hline $\mathbf{1 2}$ & 0.46 & 0.05 & 0.55 \\
\hline $\mathbf{1 3}$ & 0.70 & 0.08 & 0.91 \\
\hline $\mathbf{1 4}$ & 0.02 & 0.01 & 0.07 \\
\hline $\mathbf{1 5}$ & 0.18 & 0.00 & 0.15 \\
\hline $\mathbf{1 6}$ & 0.38 & 0.20 & 0.30 \\
\hline $\mathbf{1 7}$ & 0.16 & 0.05 & 0.12 \\
\hline $\mathbf{1 8}$ & 0.30 & 0.06 & 0.30 \\
\hline $\mathbf{1 9}$ & 0.13 & 0.05 & 0.13 \\
\hline $\mathbf{2 0}$ & 0.08 & 0.08 & 0.12 \\
\hline
\end{tabular}

Table 12. Standard deviation calculation EinScan-Pro - Artec Eva 


\begin{tabular}{|c|c|c|c|}
\hline \multicolumn{4}{|c|}{ Nikon - EinScan - Artec $[\mathbf{m m}]$} \\
\hline Points & $\sigma_{\mathrm{x}, \mathrm{G}}$ & $\sigma_{\mathrm{y}, \mathrm{G}}$ & $\sigma_{\mathrm{z}, \mathrm{G}}$ \\
\hline $\mathbf{1}$ & 1.99 & 0.95 & 0.62 \\
\hline $\mathbf{2}$ & 2.11 & 1.02 & 0.66 \\
\hline $\mathbf{3}$ & 1.08 & 0.55 & 0.08 \\
\hline $\mathbf{4}$ & 0.68 & 0.01 & 0.78 \\
\hline $\mathbf{5}$ & 0.48 & 0.20 & 0.53 \\
\hline $\mathbf{6}$ & 1.04 & 0.36 & 1.14 \\
\hline $\mathbf{7}$ & 0.51 & 0.05 & 0.27 \\
\hline $\mathbf{8}$ & 4.82 & 0.61 & 5.42 \\
\hline $\mathbf{9}$ & 0.47 & 3.50 & 1.10 \\
\hline $\mathbf{1 0}$ & 0.45 & 0.05 & 0.61 \\
\hline $\mathbf{1 1}$ & 0.41 & 0.02 & 0.38 \\
\hline $\mathbf{1 2}$ & 0.39 & 0.07 & 0.46 \\
\hline $\mathbf{1 3}$ & 0.89 & 0.28 & 0.66 \\
\hline $\mathbf{1 4}$ & 0.71 & 0.23 & 0.48 \\
\hline $\mathbf{1 5}$ & 0.44 & 0.32 & 0.34 \\
\hline $\mathbf{1 6}$ & 2.10 & 1.59 & 2.50 \\
\hline $\mathbf{1 7}$ & 0.63 & 0.46 & 0.76 \\
\hline $\mathbf{1 8}$ & 0.28 & 0.18 & 0.28 \\
\hline $\mathbf{1 9}$ & 1.61 & 1.14 & 1.82 \\
\hline $\mathbf{2 0}$ & 0.16 & 0.19 & 0.88 \\
\hline
\end{tabular}

Table 13. Global standard deviation calculation

Finally, (Table 13) the models obtained respectively with Artec Eva, Nikon D3200 and EinScan-Pro, have been compared to each other, obtaining, in this case, a limit value of $2.11 \mathrm{~mm}$ and $90 \%$ of tolerable values. It is important to underline that, although the results obtained from the error theory are satisfactory, for the photogrammetric survey the number of tolerable deviations are a little lower than that highlighted in the approach with EinScan-Pro; this is also determined by the fact that the photogrammetric model is made up of a much smaller number of triangles (100670 polygons) a consequence, certainly, of photographs taken expeditiously and not for the generation of a real photogrammetric model.

\section{CONCLUSION}

The aim of the paper was to identify a methodology of analysis for the sculptural heritage, with an empirical approach, that is conducted on the evidence on practical cases; in particular, the focus was on testing the quality characteristics of the new, entry level, EinScan-Pro scanner in the museum. It should be noted that some sculptures have characteristics that are not suitable for acquisitions with structured light scanners (dark and homogeneous textures on simple shapes), which inevitably led to the loss of signal and difficulty in aligning the different scans. The conclusions allows us to observe, in general, that the use of hand-held scanners brings with it some problems related to the operating principles on which the functioning of the instrument is based.

The structured light detection technique is easy to use, but subject to some interferences (possible effect of low contrast in strong lighting conditions typical of outdoor environments, presence of shadows due to differences in lighting typical of indoor environments and characteristics of the materials to be acquired), which is why it is very important to create a scene with characteristics suitable for the instrument and found optimal detection distance to limit random errors. Moreover, during the acquisition phase, it is necessary to pay great attention also to the movement around the object, trying to avoid sudden movements to avoid the loss of signal, with a consequent increase of time in the post-processing phase, as well as, redundant models with a number of superfluous polygons in the final meshes. In the case of EinScan-Pro the loss of signal and, inevitably, the breakdown of the acquisitions into several phases has generated models with a greater number of polygons, but often qualitatively lower.

Looking at the three-dimensional models returned by the two scanners, in fact, it is evident that EinScan-Pro, despite having metric deviations around the $\mathrm{mm}$ compared to the reference scanners, allows to obtain an even lower level of detail of the object and with a texture that is not always 'clear'. It is no coincidence that the acquisition pattern has presented greater difficulties in detecting objects with dark surfaces, areas in shadow or near corners, with the consequent presence of information gaps in the returned models. Another problem of these instruments is the already known issue of texture: the presence of cameras with limited resolution, in fact, does not allow to obtain models graphically comparable to photogrammetric models, which in the present state have a better graphic quality (Figure 10).

As can be deduced, therefore, the new low-cost hand-held technologies and here object of studies, still present some limitations. It can be deduced, therefore, that a complementary and integrated approach between the different methodologies is still today the most effective procedure, above all in the cultural heritage sector where each situation represents a case in itself, each with its own characteristics, which often force us to abandon standardized workflows to identify new and diversified solutions. However, in this direction, to know the limits and potential of the devices available is fundamental in order to achieve the objectives set.
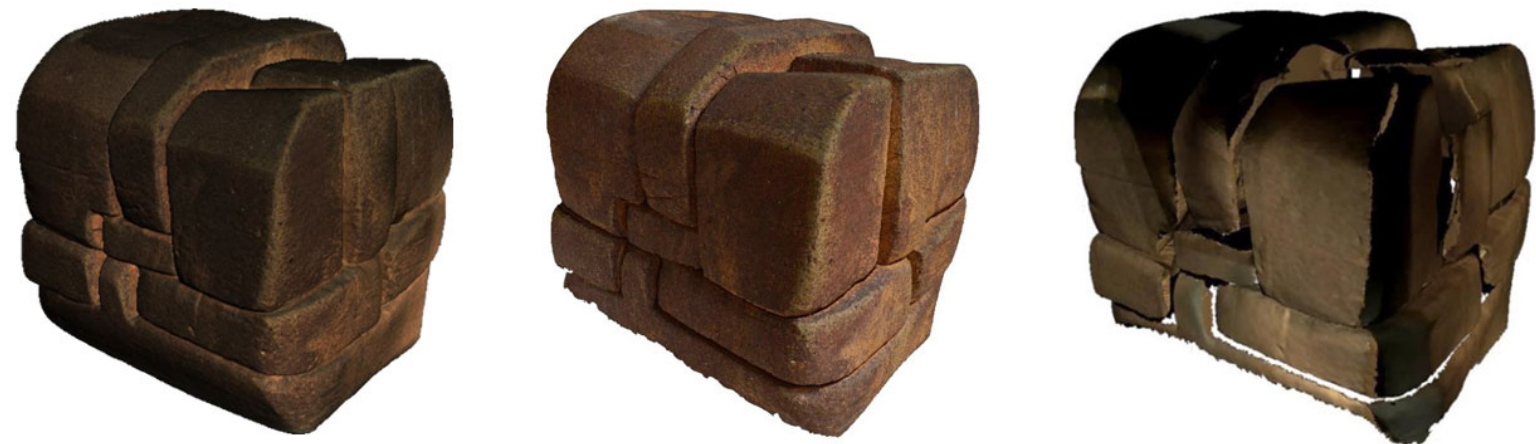

Figure 10. From left to right: model made with Artec Eva, Nikon D3200 and EinScan-Pro 


\section{ACKNOWLEDGEMENTS}

The authors acknowledge the 3D TARGET Company for the EinScan-Pro instrumentation made available to us and Maria Assunta Mammone for her collaboration in the data acquisition and post-processing.

\section{REFERENCES}

Adams, J. W., Olah, A., McCurry, M. R. \& Potze, S., 2015. Surface Model and Tomographic Archive of Fossil Primate and Other Mammal Holotype and Paratype Specimens of the Ditsong National Museum of Natural History, Pretoria, South Africa. PloS one, 1-14. doi:0.1371/journal.pone.0139800.

Akca, D., Remondino, F., Novák, D., Hanusch, T., Schrotter, G., Gruen, A. 2006. Recording and modeling of cultural heritage objects with coded structured light projection systems. 2nd International Conference on Remote Sensing in Archaeology, 375-382.

Barba, S., Mage, M.A., 2014. Evaluación ex-ante y ex-post de la precisión de un proyecto fotogramétrico. $V$ Congreso Internacional de Expresión Gráfica en Ingeniería, Arquitectura y Carreras afines, EGraFIA, 548-557.

Bohem, J., 2014. Accuracy Investigation for structured-light Based Consumer 3D Sensors. PhotogrammetrieFernerkundung - Geoinformation, (2), 117-127.

Farina, I., Goodall, R., Hernández-Nava, E., di Filippo, A., Colangelo, F. \& Fraternali, F., 2019. Design, microstructure and mechanical characterization of Ti6Al4V reinforcing elements for cement composites with fractal architecture. Materials \& Design, 172, 107758.

Garcìa Marcos, J-A., 2005. Eduardo Chillida. Editorial Txertoa.

Georgopoulos, A., Ioannidis, C., Valanis, A., 2010. Assessing the performance of a structured light scanner. ISPRS Ann. Photogramm. Remote Sens. Spatial Inf. Sci., XXXVIII-5, 250-255. doi: org/10.6084/m9.figshare.3188950.

Hayes, B. E., 1998. Measuring customer satisfaction: Survey design, use, and statistical analysis methods. Wisconsin: ASQC Quality Press.

Hess, A., 2012. Nikon D3200 Digital Field Guide. Wiley.

Inzerillo, L., Di Mino, G., Di Paola, F. \& Noto, S., 2015. The Diagnostics of Road Surface Distresses Through Image-Based Modeling Techniques. Experimental Survey on LaboratoryRutted Samples. Life Safety and Security, 3 (8), 31-35.

Jain, S., Zhang, B., 2019. Evaluation of the spatial frequency response and the uncertainty for a commercial structured light system. Proc. of SPIE: Applied Optical Metrology III Vol. 11102. doi: $10.1117 / 12.2528291$.

Kersten, T. P., Przybilla, H.-J., Lindstaedt, M., Tschirschwitz, M., Misgaiski-Hass, M., 2016. Comparative geometrical investigations of hand-held scanning systems. Int. Arch. Photogramm. Remote Sens. Spatial Inf. Sci., XLIB5, 507-514.
Laurențiu-Marian, A., 2019: Practical guide and applications of photogrammetry in cultural heritage $3 \mathrm{~d}$ digitization. Editura MEGA Nature, Cluj-Napoca.

Matos Castaño, B., "Eduardo Chillida, arquitecto", Tesi di Dottorato, Universidad Politécnica de Madrid.

Morena, S., Barba, S., Álvaro-Tordesillas, A., 2014. Fotogrammetria e tecnologia IR per un rilievo digitale lowcost della scultura "Torso", di Eduardo Chillida. AA.VV. LDL-C: Levantamiento Digital Low-Cost, Valladolid. Ediciones Universidad De Valladolid, 67-80.

Mousavi, V., Khosravi, M., ahmadi, M., Noori, N., Hosseini naveh A., Varshosaz, M., 2015. The performance evaluation of multi-image $3 \mathrm{~d}$ reconstruction software with different sensors. Int. Arch. Photogramm. Remote Sens. Spatial Inf. Sci., XL-1/W5, 515-519.

Wang, P., Jin, X., Li, S., Wang, C., Zhao, H., 2018. Digital Modeling of Slope Micro-geomorphology Based on Artec Eva 3D Scanning Technology. IOP Conf. Series: Earth and Environmental Science 252. doi:10.1088/1755$1315 / 252 / 5 / 052116$. 